\title{
On Orthogonality-preserving Plücker transformations of Hyperbolic Spaces
}

\author{
Klaus List
}

July 29, 1999

\begin{abstract}
A complete overview of all orthogonality-preserving Plücker transformations in finite dimensional hyperbolic spaces with dimension other than three is given. In the Cayley-Klein model of such a hyperbolic space all Plücker transformations are induced by collineations of the ambient projective space.
\end{abstract}

\section{Introduction}

Let $G$ be an arbitrary non-empty set and $\sim$ a binary relation on $G$ which is symmetric and reflexive. Following [3] we call the structure $(G, \sim)$ a Plücker space, if for each pair $a, b \in G$ there exists a finite number of elements $c_{1}, c_{2}, \ldots, c_{n} \in G$ with

$$
a \sim c_{1} \sim c_{2} \sim \ldots \sim c_{n} \sim b .
$$

A Plücker transformation of $(G, \sim)$ is a bijection $\varphi: G \rightarrow G$ with

$$
a \sim b \Longleftrightarrow a^{\varphi} \sim b^{\varphi} \text { for all } a, b \in G .
$$

All such Plücker transformations form the Plücker group of $(G, \sim)$.

Orthogonality-preserving Plücker transformations of Euclidean spaces have been discussed by W. BENZ and E.M. SchröDER in $[3,4]$. Here $G$ is the set of lines and $a \sim b$ means that either $a=b$ or that $a$ and $b$ meet orthogonally. Similar results for elliptic and symplectic spaces are due to H. HAVLICEK [10, 11]. One result of all these papers is that dimension three is, in some sense, exceptional. For example the 3-dimensional elliptic spaces are the only ones with Plücker transformations not induced by collineations and dualities [10]. More examples of Plücker spaces can be found in [3, 5, 13, 15].

In this paper we discuss orthogonality-preserving Plücker transformations of finite dimensional hyperbolic spaces with a Euclidean ground field. For dimension other than three all Plücker transformations are determined. In the Cayley-Klein model they are induced by collineations of the ambient projective space. Moreover, condition (1) can be reduced to

$$
a \sim b \Longrightarrow a^{\varphi} \sim b^{\varphi} \quad \forall a, b \in G .
$$




\section{$2 \quad$ Plücker spaces on hyperbolic spaces}

Let $\Pi:=\Pi(\mathcal{P}, \mathcal{G})$ be a Pappian projective space $(2 \leq \operatorname{dim} \Pi:=n<\infty)$ with point set $\mathcal{P}$, line set $\mathcal{G}$ and Euclidean ground field $\mathbb{K}$. The characteristic of a Euclidean field is always 0. Moreover, the set $\mathcal{H}$ of internal points of an oval quadric $\mathcal{Q}$ in $\Pi$ never is empty $\left[2\right.$, p.54]. Now the linear space $\Pi_{h}(\mathcal{H}, \overline{\mathcal{G}})$ with

$$
\overline{\mathcal{G}}:=\{\bar{g}=g \cap \mathcal{H} \mid \bar{g} \neq \varnothing, g \in \mathcal{G}\}
$$

is the Cayley-Klein model of the $n$-dimensional hyperbolic space over $\mathbb{K}$; cf. [8] or [14] for an axiomatic approach. We call $\mathcal{Q}$ the absolute quadric and denote its polarity by $\pi$. Since $\mathbb{K}$ is Euclidean, each hyperbolic line $\bar{g}$ has two ideal points $\{A, B\}:=g \cap \mathcal{Q}[2$, p.54]. We define a mapping of the lattice of subspaces onto itself by setting

$$
\mathcal{U} \mapsto \bigcap\left\{P^{\pi} \mid P \in \mathcal{U}\right\} \text { for all subspaces } \mathcal{U} \neq \varnothing \text { and } \varnothing \mapsto \Pi \text {. }
$$

This mapping is again denoted by $\pi$.

Since the ground field $\mathbb{K}$ of $\Pi$ is Euclidean, it can be ordered uniquely. Therefore only one separation function can be defined on $\mathcal{P}$ [2, p.60]. Pairs $A, B$ and $C, D$ with $A, B \neq C, D$ on a line or a conic are called separated, denoted by $(A, B \mid C, D)=-1$, if and only if the cross-ratio $C R(A, B, C, D)<0$. Otherwise we write $(A, B \mid C, D)=1$. Two coplanar hyperbolic lines with ideal points $A, B$ and $C, D$ intersect in $\mathcal{H}$ if and only if $(A, B \mid C, D)=-1$ [2, p.62ff].

In the following we will distinguish between a secant $g \in \mathcal{G}$ of $\mathcal{Q}$ and the hyperbolic line $\bar{g}:=g \cap \mathcal{H}$.

The polarity $\pi$ gives rise to the following binary relations $\sim$ and $\approx$ on $\overline{\mathcal{G}}$ : For $\bar{a}, \bar{b} \in \overline{\mathcal{G}}$ put

$$
\begin{array}{ll}
\bar{a} \approx \bar{b}: \Longleftrightarrow a \cap b^{\pi} \neq \varnothing \text { and } \bar{a} \cap \bar{b} \neq \varnothing & \text { (orthogonally intersecting lines) } \\
\bar{a} \sim \bar{b}: \Longleftrightarrow \bar{a} \approx \bar{b} \text { or } a=b & \text { (related lines). }
\end{array}
$$

Both relations are symmetric and, by definition, $\sim$ is reflexive. Now we can show:

Proposition 1. The structure $(\overline{\mathcal{G}}, \sim)$ is a Plücker space.

Proof. Let $\bar{a}, \bar{b} \in \overline{\mathcal{G}}$ be distinct. First we assume that $a, b$ are in a plane $\varepsilon$ with $\mathcal{Q}_{\varepsilon}:=\mathcal{Q} \cap \varepsilon$. We are looking for a finite sequence of lines $\bar{n}_{1}, \bar{n}_{2}, \ldots, \bar{n}_{k} \in \overline{\mathcal{G}}$ with

$$
\bar{a} \sim \bar{n}_{1} \sim \ldots \sim \bar{n}_{k} \sim \bar{b}
$$

1. For hyperparallel lines $\bar{a}, \bar{b}$, the intersection point $a \cap b$ is an external point. Hence the line $n:=(a \cap b)^{\pi} \cap \varepsilon$ fulfills $\bar{a} \sim \bar{n} \sim \bar{b}$.

2. Now let $\bar{a}, \bar{b}$ be parallel and $A \in a \cap \mathcal{Q}_{\varepsilon}, B \in b \cap \mathcal{Q}_{\varepsilon}$ and $C:=a \cap b \in$ $\mathcal{Q}_{\varepsilon}$ with $A \neq C \neq B$. Furthermore we choose $D, E \in \mathcal{Q}_{\varepsilon}$ with $D \neq$ $E$ such that the pairs $(A, D),(C, B)$ and $(A, E),(C, B)$ are separated. From $(C, B \mid D, A)=-1,(C, B \mid A, E)=-1,(A, C \mid D, B)=1$, and 
$(A, C \mid B, E)=1$ the multiplication theorem for separation functions gives $(C, B \mid D, E)=1$ and $(A, C \mid D, E)=1$. So the line $\bar{n}_{2}:=D E \cap \mathcal{H}$ is hyperparallel to $\bar{a}$ and $\bar{b}$. Now we have reduced the problem to case 1 .

3. $\bar{a}, \bar{b}$ intersect: We choose $B$ in $b \cap \mathcal{Q}_{\varepsilon}$. Then $\bar{n}_{1}:=\left(\left(a^{\pi} \cap \varepsilon\right) B\right) \cap \mathcal{H}$ is parallel to $\bar{b}$ and $\bar{a} \sim \bar{n}_{1}$. Again, we have reduced the problem to case 2 .

If $a, b$ are skew lines we choose a line $\bar{c}$ intersecting $\bar{a}$ and $\bar{b}$. So the assertion follows from case 3 . Any two lines in $\overline{\mathcal{G}}$ are either hyperparallel, or parallel, or intersecting, or skew.

In the proof of Proposition 1 we saw that for any two skew lines $\bar{a}, \bar{b} \in \overline{\mathcal{G}}$ there exists a sequence of orthogonally intersecting lines that connect $\bar{a}$ and $\bar{b}$. By $[7$, p.64, (1)] it is always possible to reduce this sequence to one line:

Lemma 1. Let $\bar{a}, \bar{b}$ be two given skew lines of a hyperbolic space $\Pi_{h}$. Then there exists a line $\bar{n}$ intersecting $\bar{a}$ and $\bar{b}$ orthogonally.

Now we want to discuss Plücker transformations of $(\overline{\mathcal{G}}, \sim)$. We use the term $\mathcal{Q}$-collineation for any collineation of $\Pi$ leaving the quadric $\mathcal{Q}$ invariant. It is obvious that $\mathcal{Q}$-collineations induce Plücker transformations:

Proposition 2. Let $\psi: \Pi \rightarrow \Pi$ be a $\mathcal{Q}$-collineation. Then the line mapping $\varphi: \overline{\mathcal{G}} \rightarrow \overline{\mathcal{G}}, \bar{g} \mapsto g^{\psi} \cap \mathcal{H}$ which is induced by $\psi$ is a Plücker transformation of $(\overline{\mathcal{G}}, \sim)$.

Now the question is the following: Are all Plücker transformations induced by $\mathcal{Q}$-collineations.

\section{Plücker transformations in hyperbolic spaces with $\operatorname{dim} \Pi_{h} \geq 4$}

Let $\Pi_{h}(\mathcal{H}, \overline{\mathcal{G}})$ be a hyperbolic space $\left(\operatorname{dim} \Pi_{h} \geq 2\right)$ with the relations $\sim$ and $\approx$.

Lemma 2. Given mutually distinct $\bar{a}, \bar{b}, \bar{c} \in \overline{\mathcal{G}}$ with $\bar{a}, \bar{b}$ concurrent and $\bar{c}$ intersecting $\bar{a}$ and $\bar{b}$ orthogonally, then $\bar{a} \cap \bar{b} \subset \bar{c}$.

Proof. Since $\bar{a}, \bar{b}, \bar{c}$ are mutually distinct, $|\bar{a} \cap \bar{b}|=|\bar{a} \cap \bar{c}|=|\bar{b} \cap \bar{c}|=1$. In $\Pi_{h}$ there exists no triangle with two right angles. Therefore the point $\bar{a} \cap \bar{b}$ is on $\bar{c}$.

Proposition 3. Let $\operatorname{dim} \Pi_{h} \geq 4$ and $\varphi: \overline{\mathcal{G}} \rightarrow \overline{\mathcal{G}}$ be a bijection ${ }^{1}$ with

$$
\bar{a} \sim \bar{b} \Longrightarrow \bar{a}^{\varphi} \sim \bar{b}^{\varphi} \quad \forall \bar{a}, \bar{b} \in \overline{\mathcal{G}} .
$$

Then for every point $A \in \mathcal{H}$ there exists an $A^{\prime}$ such that $\bar{g} \in \mathcal{G}$ and $A \in \bar{g}$ implies $A^{\prime} \in \bar{g}^{\varphi}$.

\footnotetext{
${ }^{1}$ The mapping $\varphi$ may also be seen as a bijection on secants $s \in \mathcal{G}$. By abuse of notation, we define the line $s^{\varphi}$ as the unique projective line such that $\bar{s}^{\varphi}=s^{\varphi} \cap \mathcal{H}$.
} 
Proof. We choose $\bar{a}, \bar{b} \in \overline{\mathcal{G}}$ with $\bar{a} \cap \bar{b}=A$ and $\bar{a} \approx \bar{b}$. Hence $\bar{a}^{\varphi} \approx \bar{b}^{\varphi}$ and we are able to define $A^{\prime}:=\bar{a}^{\varphi} \cap \bar{b}^{\varphi}$. Now it remains to be shown that ${ }^{2} \bar{g} \in \overline{\mathcal{G}}_{A}$ implies $\bar{g}^{\varphi} \in \overline{\mathcal{G}}_{A^{\prime}}$.

1. If $\bar{g}$ is related to $\bar{a}$ and $\bar{b}$, then $\bar{a}^{\varphi} \sim \bar{g}^{\varphi} \sim \bar{b}^{\varphi}$. By Lemma $2, A^{\prime} \in \bar{g}^{\varphi}$.

2. Let $\bar{g}$ be not related to $\bar{a}$ and $\bar{b}$. Since $\operatorname{dim} \Pi_{h}=n \geq 4$, all lines passing $A$, and orthogonal to $\bar{a}$ and $\bar{b}$, span a subspace $\beta$ of $\Pi$ of dimension $n-2 \geq 2$. We put $a \vee b=: \alpha$ and choose $c, d \in \beta$ such that $\bar{c} \approx \bar{d}$ and $A \in c, d$ (see Figure 1). Additionally, there exist lines $e, f \ni A$ with $e \in \alpha, f \in \beta$ and $\bar{e} \approx \bar{g}, \bar{f} \approx \bar{g}$. Now the lines $\bar{a}, \bar{b}, \bar{c}, \bar{d}$ and $\bar{e}, \bar{f}, \bar{g}$ as well as $\bar{a}, \bar{b}, \bar{f}$ and $\bar{c}, \bar{d}, \bar{e}$ are mutually orthogonal. This is also true for their $\varphi$-images. Together with Lemma 2 we get step by step: $A^{\prime} \in \bar{c}^{\varphi}, A^{\prime} \in \bar{d}^{\varphi}$, whence $A^{\prime} \in \bar{e}^{\varphi}, A^{\prime} \in \bar{f}^{\varphi}$, and finally $A^{\prime} \in \bar{g}^{\varphi}$.

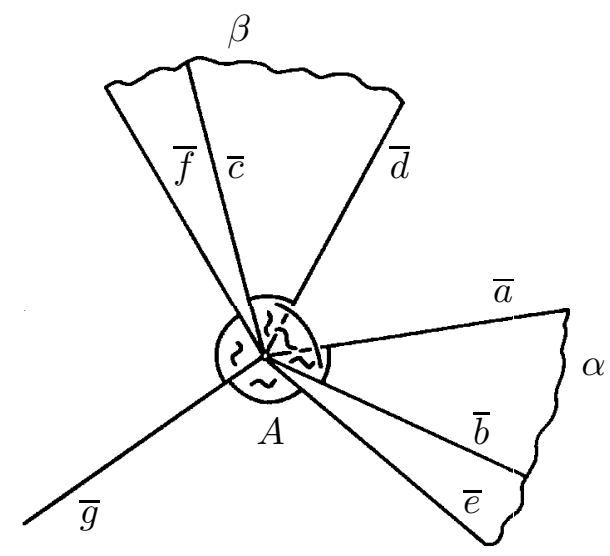

Figure 1: Step 2 of the proof of Proposition 3

In step 2 we used $\operatorname{dim} \Pi_{h} \geq 4$. Therefore, we cannot use the same methods for solving the 2- and 3-dimensional case.

With the help of $\varphi$ we are able to define a mapping $\bar{\psi}$ on the point set of $\Pi_{h}$ :

Proposition 4. Let $\Pi_{h}$ be a hyperbolic space with $\operatorname{dim} \Pi_{h} \geq 4$. If $\varphi: \overline{\mathcal{G}} \rightarrow \overline{\mathcal{G}}$ is a bijection satisfying property (3), then $\varphi$ is induced by a collineation $\bar{\psi}$ of $\Pi_{h}$.

Proof. We define $\bar{\psi}: \mathcal{H} \rightarrow \mathcal{H}, A \mapsto A^{\bar{\psi}}:=A^{\prime}$. This $\bar{\psi}$ is well defined by Proposition 3 and collinearity of points is invariant under $\varphi$.

1. Assume to the contrary that there exist two different points $A, B \in \mathcal{H}$ with $A^{\bar{\psi}}=B^{\bar{\psi}}$. For all $X \in \mathcal{H} \backslash A B$ we obtain $\overline{X B}^{\varphi} \neq \overline{X A}^{\varphi}$ by the injectivity

\footnotetext{
${ }^{2}$ By $\overline{\mathcal{G}}_{A}$ we denote the star of hyperbolic lines centered in $A$.
} 
of $\varphi$. Further $X^{\bar{\psi}}=A^{\bar{\psi}}=B^{\bar{\psi}}$. So, for every $\bar{g} \in \overline{\mathcal{G}}$ we get $A^{\bar{\psi}} \in \bar{g}^{\varphi}$, which is a contradiction to the surjectivity of $\varphi$. Thus the mapping $\bar{\psi}$ is injective.

2. Let the points $A, B, C \in \mathcal{H}$ be non-collinear and let their images $A^{\bar{\psi}}, B^{\bar{\psi}}$, $C^{\bar{\psi}}$ be on a line $\bar{g}$. Since $\bar{\psi}$ is injective, these points are mutually distinct. Now $\overline{A B} \neq \overline{A C}$ and

$$
\overline{A B}^{\varphi}=\overline{A^{\bar{\psi}} B^{\bar{\psi}}}=\bar{g}=\overline{A^{\bar{\psi}} C^{\bar{\psi}}}=\overline{A C}{ }^{\varphi}
$$

is a contradiction to the injectivity of $\varphi$.

3. The surjectivity of $\bar{\psi}$ remains to be shown:

(a) First we prove that the restriction of $\varphi$ to $\overline{\mathcal{G}}_{A}(A \in \mathcal{H})$ is a bijection onto $\overline{\mathcal{G}}{ }_{A^{\bar{\psi}}}$. For any two lines $\bar{a} \neq \bar{b}$ through $A$ it follows that $\bar{a}^{\varphi} \neq \bar{b}^{\varphi}$ and so $A^{\bar{\psi}}=\bar{a}^{\varphi} \cap \bar{b}^{\varphi}$. Supposing $A^{\bar{\psi}} \in \bar{c}^{\varphi}$ but $A \notin \bar{c}$, we can also assume without loss of generality that $\bar{c}$ intersects $\bar{a}$ and $\bar{b}$. Hence $\bar{a} \cap \bar{c}=B \neq A$ and $A^{\bar{\psi}}=B^{\bar{\psi}}$. This contradicts the injectivity of $\bar{\psi}$.

(b) Now we will show that the $\varphi$-preimages of parallel lines are again parallel: If two lines are hyperparallel or skew, then they have a common orthogonal line (Proposition 1 and Lemma 1), intersecting the lines in two different points. This is also true for their images. Concurrent and parallel lines do not have such a common orthogonal line (Lemma 2). Therefore, their $\varphi$-preimages are again concurrent or parallel. In Proposition 3 we proved that $\varphi$ maps intersecting lines to intersecting lines. Hence the assertion follows.

(c) In the next step we prove that

$$
\bar{a}^{\bar{\psi}}=\left\{X^{\bar{\psi}} \mid X \in \bar{a}\right\}=\bar{a}^{\varphi} \text { for all } \bar{a} \in \overline{\mathcal{G}} .
$$

Let us take a look at a star with center $A \notin \bar{a}$. In (b) we saw that a line $\bar{b}$, with $\bar{b}^{\varphi} \cap \bar{a}^{\varphi} \neq \varnothing$, is necessarily parallel or concurrent to $\bar{a}$. But the only two lines in $\overline{\mathcal{G}}_{A}$ being parallel to $\bar{a}$ are the $\varphi$-preimages of the parallel lines to $\bar{a}^{\varphi}$. Therefore $\bar{a}$ and $\bar{b}$ intersect and $\bar{\psi} \mid \bar{a}: \bar{a} \rightarrow \bar{a}^{\varphi}$ is surjective.

(d) If $B^{\prime}$ is an arbitrary point in $\mathcal{H}$, then we are able to choose a line $\bar{a}^{\varphi} \ni B^{\prime}$. In (c) we proved the existence of a point $B \in \bar{a}$ with $B^{\bar{\psi}}=B^{\prime}$.

Finally, we extend the collineation $\bar{\psi}: \mathcal{H} \rightarrow \mathcal{H}$ into the ambient projective space П. The main tool will be a Theorem due to R. FRANK [6].

Proposition 5. Let $\varphi$ be a bijection satisfying (3) in a hyperbolic space $\Pi_{h}$ with $\operatorname{dim} \Pi_{h} \geq 4$. Then $\varphi$ is induced by a $\mathcal{Q}$-collineation $\psi$ of $\Pi$. Moreover, $\varphi$ is a Plücker transformation. 
Proof. We already know that $\varphi$ is induced by a collineation $\bar{\psi}: \mathcal{H} \rightarrow \mathcal{H}$. Using the terminology of [6], such a collineation can be extended to a projection $\bar{\psi}$ : $\mathcal{H} \rightarrow \Pi$.

The Euclidean ground field $\mathbb{K}$, together with the order topology, is a topological field [17]. So $\Pi$ becomes a topological projective space with the coordinate topology $\tau$ [16]. The set of internal points of any oval quadric, for example $\mathcal{H}$, is an open set of $\tau$. Since $\mathcal{H}$ is not contained in a hyperplane, $\operatorname{span} \mathcal{H}^{\bar{\psi}}=\operatorname{span} \mathcal{H}=\Pi$. The induced topologies on the lines of $\Pi$ form a linear topology in the sense of [6]. Because $\mathcal{H}$ is an open set, the intersection set of every line $g$ with $\mathcal{H}$ is an open set with respect to the induced topology on $g$. So $\mathcal{H}$ is linearly open. If $\bar{g}$ is a hyperbolic line, then $\bar{g} \bar{\psi}=(\mathcal{H} \cap g)^{\bar{\psi}}=\mathcal{H} \cap g^{\varphi} \neq \varnothing$ is again open with respect to the linear topology of $\Pi$. Therefore we can use Theorem 2 of [6]:

There exist subspaces $Z \subset \Pi \backslash \mathcal{H}$ and $D \subset \Pi \backslash Z$ with span $D \vee Z=\Pi$. Moreover there exists a collineation $\psi: D \rightarrow D^{\prime}$ with $\bar{\psi}=p \psi \iota$ where $p: \mathcal{H} \rightarrow D$ is a central projection with center $Z, D^{\prime}$ is a subspace of $\Pi$ and $\iota: D^{\prime} \rightarrow \Pi$ is the canonical injection. In our case $Z$ is empty, otherwise each hyperbolic line $\bar{g}$ with $g \cap Z \neq \varnothing$ would be mapped onto a point. Furthermore, span $D \vee Z=\Pi$ implies $D=\Pi=D^{\prime}$ and $\iota$ is the identity. Hence the central projection $p: \mathcal{H} \rightarrow \Pi$ is the canonical injection and $\psi \mid \mathcal{H}=\bar{\psi}$.

Under the collineation $\psi$ hyperplanes are mapped onto hyperplanes. There is a one-to-one correspondence between external points $A$ of $\mathcal{Q}$ and hyperplanes $\varepsilon=A^{\pi}$ which contain internal points. If $I_{1}, I_{2} \in \varepsilon$ are two different internal points, $\varepsilon$ is spanned by all lines $a \in \mathcal{G} \cap \varepsilon$ with $\bar{a} \approx \overline{A I_{1}}, \bar{a} \approx \overline{A I_{2}}$ respectively. Orthogonality is invariant under $\psi$. That means $\overline{A^{\psi} I_{1}^{\psi}}\left(\overline{A^{\psi} I_{2}^{\psi}}\right)$ is the only line through $I_{1}^{\psi}\left(I_{2}^{\psi}\right)$, that is orthogonal to all lines of the star with center $I_{1}^{\psi}\left(I_{2}^{\psi}\right)$ in $\varepsilon^{\psi}$. Therefore $A^{\psi}=\varepsilon^{\psi \pi}$ is an external point. Since $\psi$ and $\bar{\psi}$ are collineations, $\psi$ yields also a bijection on the set of external points of $\mathcal{Q}$. So, $\psi$ is a $\mathcal{Q}$-collineation. Proposition 2 shows that $\varphi: \overline{\mathcal{G}} \rightarrow \overline{\mathcal{G}}$ is a Plücker transformation.

Remark. For real hyperbolic spaces we could show Proposition 5 also with Theorem 2 in [12] by R. HöFER.

\section{The 2-dimensional case}

\subsection{A characterization of Plücker transformations}

If $\operatorname{dim} \Pi_{h}=2$, then the absolute quadric $\mathcal{Q}$ is a conic with polarity $\pi$. For an arbitrary line $\bar{g} \in \overline{\mathcal{G}}$ all orthogonal lines are running through the point $g^{\pi}$. By $\operatorname{dim} \Pi_{h}=2$, orthogonal hyperbolic lines are intersecting. Therefore there exists no common orthogonal transversal for $\bar{a}, \bar{b} \in \overline{\mathcal{G}}$ being parallel or concurrent. But the sequence (2) of related lines connecting $\bar{a}, \bar{b}$ can be reduced to two lines $\overline{n_{1}}, \overline{n_{2}}$ : 
Lemma 3. In a hyperbolic plane let $\bar{a}, \bar{b} \in \overline{\mathcal{G}}$ be two different lines, that are parallel or intersecting, but not orthogonal. Then there exist $\overline{n_{1}}, \overline{n_{2}} \in \overline{\mathcal{G}}$ with

$$
\bar{a} \approx \overline{n_{1}} \approx \overline{n_{2}} \approx \bar{b}
$$

Proof. In both cases we will show the existence of a line $\overline{n_{1}}$ with $\bar{a} \approx \overline{n_{1}}$ that is hyperparallel to $\bar{b}$.

1. $\bar{a}, \bar{b}$ are parallel: Let $A \in a \cap \mathcal{Q}, B \in b \cap \mathcal{Q}$ and $C:=a \cap b \in \mathcal{Q}$ with $A \neq C \neq B$. There exists a $D \in \mathcal{Q}$ such that the pairs $(A, B)$ and $(C, D)$ are seperated. The line $a^{\pi} D$ meets $\mathcal{Q}$ residually at a point $E$, say. Then $(A, C \mid D, E)=-1$, because $\overline{A C}$ and $\overline{D E}$ intersect orthogonally. Thus

$$
(C, D \mid B, E)=(C, D \mid B, A) \cdot(C, D \mid A, E)=(-1) \cdot 1=-1
$$

and $(B, C \mid D, E)=1$, which means, $\bar{b}$ and $\overline{n_{1}}:=\overline{D E}$ are hyperparallel.

2. $\bar{a}, \bar{b}$ are intersecting (Figure 2): Let $A \neq B$ and $C \neq D$ be the intersection points of $a$ and $b$ with $\mathcal{Q}$. Because $\bar{a}, \bar{b}$ intersect, $(A, B \mid C, D)=-1$. Choose $E \in a^{\pi} D \cap \mathcal{Q}$ with $D \neq E$. So the lines $\overline{A B}$ and $\overline{D E}$ intersect, i.e. $(A, B \mid D, E)=-1$. From $a \not \psi b$ follows $C \neq E$. Without loss of generality we can assume that $(A, C \mid D, E)=-1$ : If $(A, C \mid D, E)=1$ then the multiplication theorem for seperation functions gives:

$$
(B, C \mid D, E)=(B, A \mid D, E) \cdot(A, C \mid D, E)=(-1) \cdot 1=-1 .
$$

Moreover, we choose $F$ such that $(A, D \mid C, F)=-1$. For the second intersection point $G \in a^{\pi} F \cap \mathcal{Q}$ we get $(E, D \mid F, G)=1$, since $E D, F G$ intersect in the external point $a^{\pi}$ of $\mathcal{Q}$. Now we get step by step:

$$
\begin{aligned}
(A, D \mid E, F) & =(A, D \mid C, F) \cdot(A, D \mid E, C)=(-1) \cdot 1=-1, \\
(D, E \mid A, G) & =(D, E \mid F, G) \cdot(D, E \mid A, F)=1 \cdot 1=1, \\
(D, E \mid C, G) & =(D, E \mid A, G) \cdot(D, E \mid C, A)=1 \cdot(-1)=-1, \\
(D, E \mid C, F) & =(D, E \mid C, G) \cdot(D, E \mid G, F)=(-1) \cdot 1=-1 .
\end{aligned}
$$

Thus $(C, D \mid F, G)=(C, D \mid E, G) \cdot(C, D \mid F, E)=1 \cdot 1=1$ and therefore $\bar{b}$ and $\overline{n_{1}}:=\overline{G F}$ are hyperparallel.

Proposition 6. In every hyperbolic plane $\Pi_{h}$ a bijection

$$
\varphi: \overline{\mathcal{G}} \rightarrow \overline{\mathcal{G}} \text { with } \bar{g} \sim \bar{h} \Longrightarrow \bar{g}^{\varphi} \sim \bar{h}^{\varphi}
$$

is a Plücker transformation of $(\overline{\mathcal{G}}, \sim)$.

Proof. For two arbitrary lines $\bar{g}, \bar{h} \in \overline{\mathcal{G}}$ we show

$$
\bar{g} \nsim \bar{h} \Longrightarrow \bar{g}^{\varphi} \nsim \bar{h}^{\varphi} .
$$




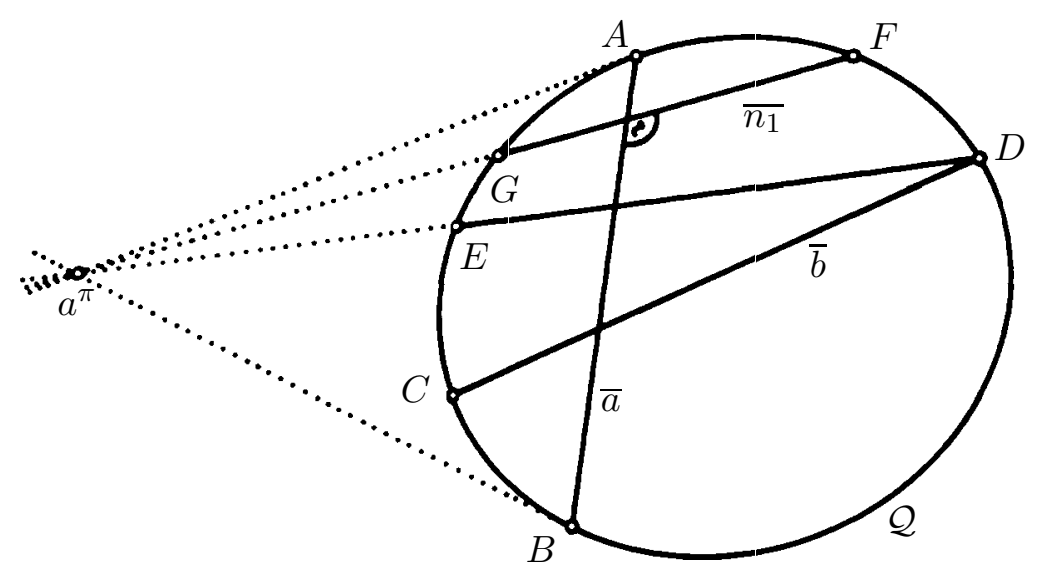

Figure 2: $\bar{a}, \bar{b}$ are intersecting

1. For every two hyperparallel lines $\bar{g}, \bar{h}$ there exists a line $\overline{n_{1}} \in \overline{\mathcal{G}}$ with $\bar{g} \approx \overline{n_{1}} \approx \bar{h}$ and furthermore $\bar{g}^{\varphi} \approx \bar{n}_{1}^{\varphi} \approx \bar{h}^{\varphi}$. Therefore $n_{1}^{\varphi \pi}=g^{\varphi} \cap h^{\varphi}$ is an external point of $\mathcal{Q}$ and $\bar{g}^{\varphi}, \bar{h}^{\varphi}$ are hyperparallel as well.

2. If $\bar{g}, \bar{h}$ are parallel or intersecting then, by Lemma 3 , there exist lines $\overline{n_{1}}, \overline{n_{2}} \in \overline{\mathcal{G}}$ with $\bar{g} \approx \overline{n_{1}} \approx \overline{n_{2}} \approx \bar{h}$. Our assumptions lead to $\bar{g}^{\varphi} \approx \bar{n}_{1}^{\varphi} \approx$ $\bar{n}_{2}^{\varphi} \approx \bar{h}^{\varphi}$. But in a hyperbolic plane there exists no rectangle.

So in both cases $\bar{g}^{\varphi} \nsim \bar{h}^{\varphi}$ is true.

In this proof the crucial point is that $\operatorname{dim} \Pi_{h}=2$. Otherwise two intersecting lines have a common orthogonal line and there is even the possibility of $\bar{g}, \bar{h}$ being skew. Therefore we cannot use the same methods for $\operatorname{dim} \Pi_{h} \geq 3$.

\subsection{Induced collineations on $\Pi_{h}$ and $\Pi$}

Together with every Plücker transformation $\varphi$ of $(\overline{\mathcal{G}}, \sim)$ we have the bijection

$$
\left(\pi \mid A_{\mathcal{Q}}\right) \varphi \pi: A_{\mathcal{Q}} \rightarrow A_{\mathcal{Q}}
$$

on the set of external points $A_{\mathcal{Q}}$. We denote this bijection again by $\varphi$. From now on let $\varphi$ be the mapping

$$
\varphi: \overline{\mathcal{G}} \cup A_{\mathcal{Q}} \rightarrow \overline{\mathcal{G}} \cup A_{\mathcal{Q}}
$$

with $\overline{\mathcal{G}}^{\varphi}=\overline{\mathcal{G}}$ and $A_{\mathcal{Q}}^{\varphi}=A_{\mathcal{Q}}$. For every $A \in A_{\mathcal{Q}}$ and every secant $g$ of $\mathcal{Q}$ there is

$$
A \in g \Longleftrightarrow \bar{g} \approx \overline{A^{\pi}} \Longleftrightarrow \bar{g}^{\varphi} \approx{\overline{A^{\pi}}}^{\varphi} \Longleftrightarrow A^{\pi \varphi \pi} \in g^{\varphi}: \Longleftrightarrow A^{\varphi} \in g^{\varphi} .
$$

Proposition 7. Assume that $\varphi$ satisfies the conditions of Proposition 6. Then for each point $A \in \mathcal{H}$ there exists an $A^{\prime} \in \mathcal{H}$ with $\overline{\mathcal{G}}_{A}^{\varphi} \subset \overline{\mathcal{G}}_{A^{\prime}}$.

Using the polarity $\pi$ we are able to translate Proposition 7 into an equivalent proposition concerning external points of $\mathcal{Q}$ : 
Proposition 8. Let $G, H$ and $I$ be three distinct points on an external line of $\mathcal{Q}$. Then there exists an external line of $\mathcal{Q}$ that contain $G^{\varphi}, H^{\varphi}$, and $I^{\varphi}$.

Proof. We will establish Proposition 8 by constructing a nontrivial Desargues configuration $Z, P_{j}, Q_{j}(j \in\{1,2,3\})$ such that corresponding edges $p_{j}, q_{j}(j \in$ $\{1,2,3\})$ meet at $G, H$ and $I$. The vertices of the triangles $P_{1}, P_{2}, P_{3}$ and $Q_{1}, Q_{2}, Q_{3}$ will be external points and the edges $p_{j}, q_{j}(j \in\{1,2,3\})$ will be secants of $\mathcal{Q}$ (see Figure 3 ).

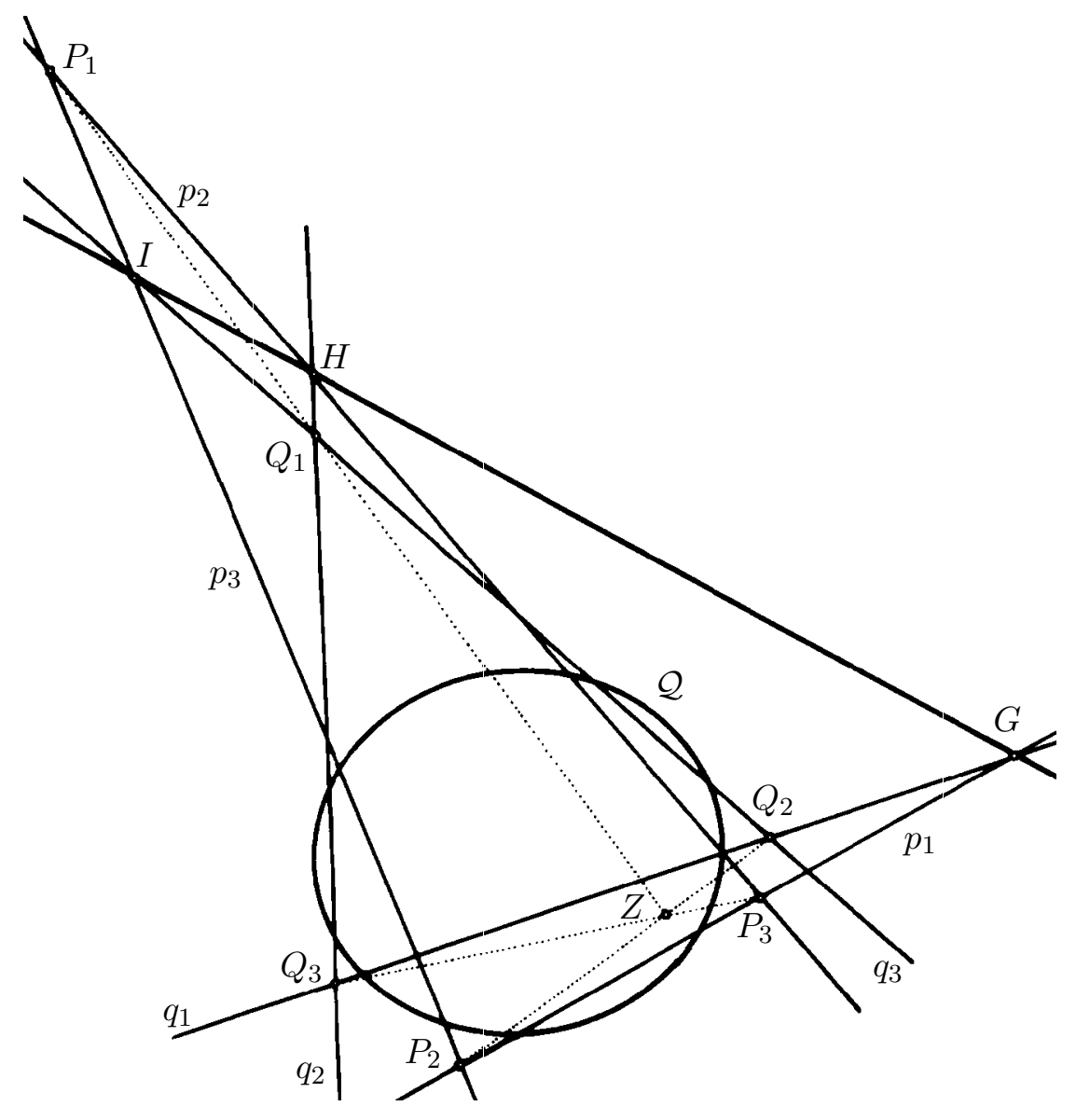

Figure 3: Desargues configuration with $P_{j}, Q_{j}$ external points and $p_{j}, q_{j}$ secants of $\mathcal{Q}(j \in\{1,2,3\})$

1. Through each point $G, H$, and $I$ we choose a tangent line of $\mathcal{Q}\left(t_{G}, t_{H}\right.$ and $\left.t_{I}\right)$. Since $u:=G H$ does not intersect $\mathcal{Q}$, the points of tangency $T_{G}, T_{H}, T_{I}$ as well as $G_{0}:=t_{H} \cap t_{I}, H_{0}:=t_{G} \cap t_{I}$ and $I_{0}:=t_{G} \cap t_{H}$ are mutually distinct and form a triangle ${ }^{3}$ (see Figure 4).

If we choose $u$ as the line at infinity, we get the affine plane $\mathcal{A}:=\mathcal{P} \backslash u$.

\footnotetext{
${ }^{3}$ Just if $G H$ is a tangent line $T_{G}=T_{H}$ is possible.
} 


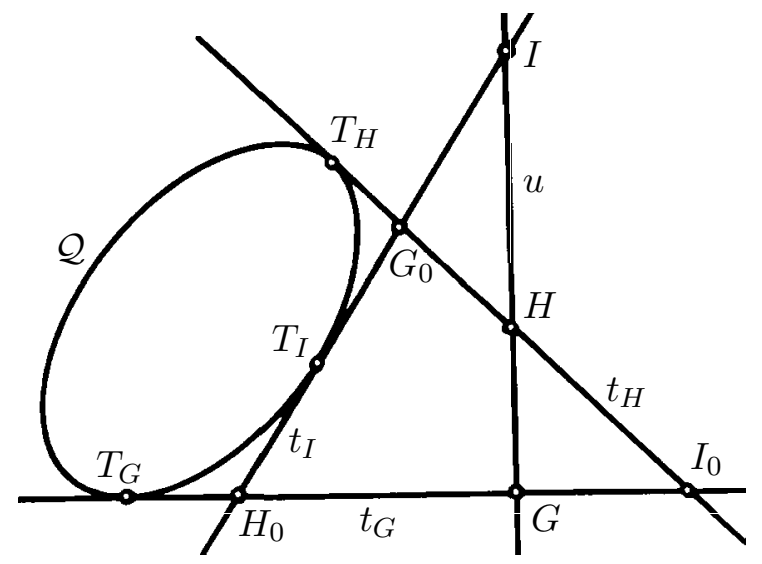

Figure 4: Step 1 of the proof of Proposition 8

We endow $\mathcal{A}$ with a $\mathbb{K}$-metric ${ }^{4}$

$$
\sigma: \mathcal{A} \times \mathcal{A} \rightarrow \mathbb{K}
$$

in the sense of S. GUDDER [9].

2. Since $G_{0}, H_{0}, I_{0}$ are external points of $\mathcal{Q}$ and $A_{\mathcal{Q}} \backslash u$ is an open set, there exists a $\rho \in \mathbb{K}$ with $\rho>0$ such that the open disks $K\left(G_{0}, \rho\right), K\left(H_{0}, \rho\right)$, and $K\left(I_{0}, \rho\right)$ are subsets of $A_{\mathcal{Q}}$. Now we construct, for example, the line $p_{1}$ : Inside the disk $K\left(T_{G}, \frac{\rho}{3}\right)$ we choose the points $G_{H}, G_{I}$ such that $G_{H} \in T_{G} H$ and $G_{I} \in T_{G} I$ are internal points of $\mathcal{Q}$ (see Figure 5).

Without loss of generality $p_{1}:=G_{H} G$ is between $t_{G}$ and $G_{I} G$. Therefore the intersection point $p_{1} \cap T_{G} I$ lies between $T_{G}$ and $G_{I}$. Furthermore $p_{1} \cap T_{G} I$ is an internal point of $K\left(T_{G}, \frac{\rho}{3}\right)$. But since $\mathbb{K}$ is a Euclidean field and $p_{1}$ has at least one internal point, $p_{1}$ is a secant of $\mathcal{Q}$. Analogously we are able to construct $p_{2}$ and $p_{3}$.

3. In the next step we will show that the three points $P_{i}:=p_{j} \cap p_{k}(\{i, j, k\}=$ $\{1,2,3\}$ ) are external points of $\mathcal{Q}$ (see Figure 6). With

$$
\sigma\left(G_{0}, p_{2} \cap G_{0} T_{I}\right)=\sigma\left(T_{H}, H_{I}\right)<\frac{\rho}{3}
$$

and

$$
\sigma\left(p_{2} \cap G_{0} T_{I}, P_{1}\right)=\sigma\left(T_{I}, I_{H}\right)<\frac{\rho}{3}
$$

we get

$$
\sigma\left(G_{0}, P_{1}\right) \leq \sigma\left(G_{0}, p_{2} \cap G_{0} T_{I}\right)+\sigma\left(p_{2} \cap G_{0} T_{I}, P_{1}\right)<\frac{\rho}{3}+\frac{\rho}{3}<\rho .
$$

\footnotetext{
${ }^{4} \mathcal{A}$ is isomorphic to the affine plane $\mathcal{A}\left(\mathbb{K}^{2}\right)$ over the field $\mathbb{K}$. $\mathcal{A}\left(\mathbb{K}^{2}\right)$ together with $\sigma$ : $\mathbb{K}^{2} \times \mathbb{K}^{2} \rightarrow \mathbb{K},\left(\left(x_{1}, x_{2}\right),\left(y_{1}, y_{2}\right)\right) \mapsto \sqrt{\left(y_{1}-x_{1}\right)^{2}+\left(y_{2}-y_{2}\right)^{2}}$ forms a so called $\mathbb{K}$-metric space fulfilling the usual conditions of a metric space. It turns out [9] that for a $\mathbb{K}$-metric space there exists a cardinal $\alpha$, such that the intersection of a family, with the cardinality less than $\alpha$, of open sets is open. Such $\alpha$-topological spaces over $\mathbb{K}$-metric spaces have a lot of properties with topological spaces over metric spaces in common. For example they are Hausdorff, and they are even normal. For a detailed description see [9].
} 


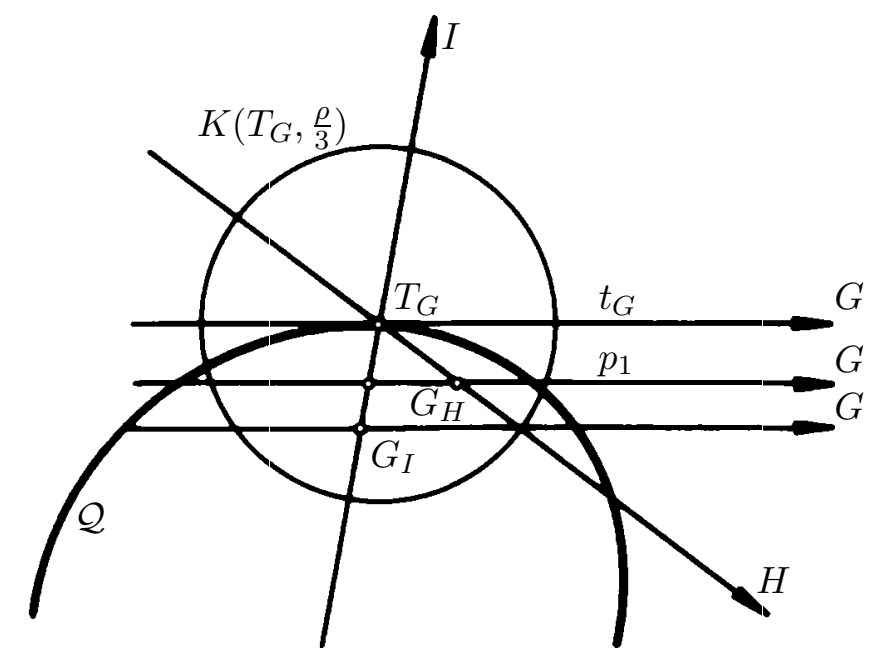

Figure 5: Step 2 of the proof of Proposition 8

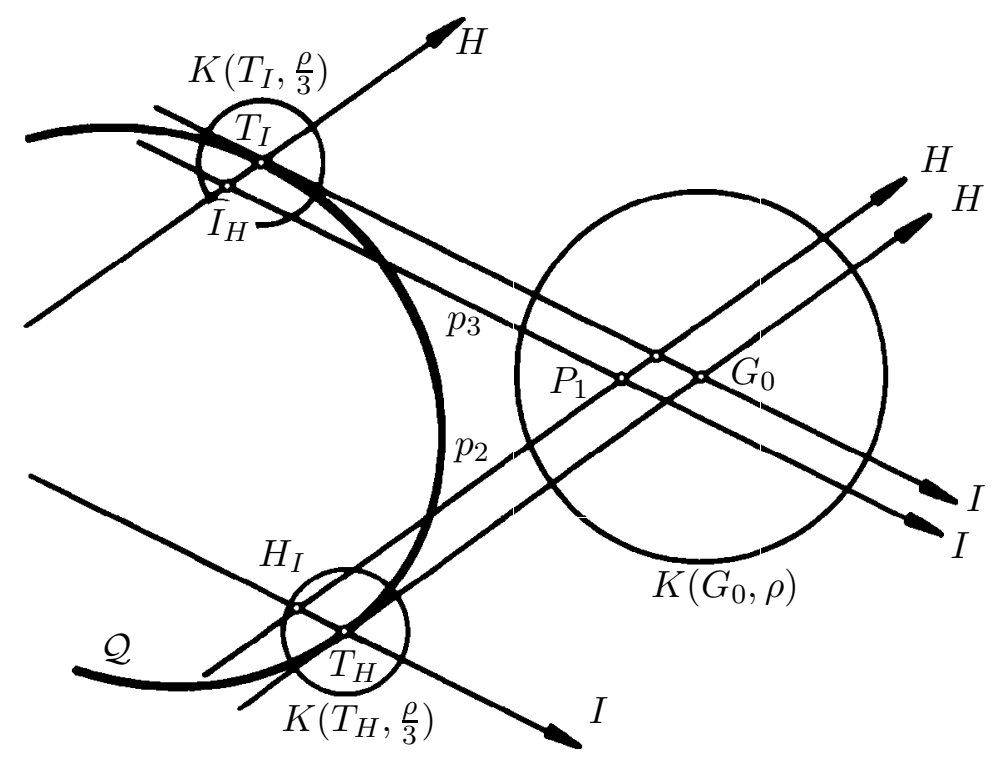

Figure 6: Step 3 of the proof of Proposition 8

Therefore $P_{1} \in K\left(G_{0}, \rho\right)$ is an external point of $\mathcal{Q}$. Analogously this can be shown for $P_{2}$ and $P_{3}$.

4. Let us choose $\widehat{\rho} \in \mathbb{K}$ with $0<\widehat{\rho}<\frac{\rho}{3}$ such that there exist points $\widehat{M}_{i} \in p_{i}$ $(i \in\{1,2,3\})$ with $K\left(\widehat{M}_{i}, \widehat{\rho}\right) \subset \mathcal{H}$. Furthermore let $Q_{1} \in K\left(P_{1}, \widehat{\rho}\right)$ neither be on $P_{1} H$ nor on $P_{1} I$ and let $\tau: \mathcal{A} \rightarrow \mathcal{A}$ be the translation with $\tau\left(P_{1}\right)=$ $Q_{1}$. Then $Q_{1}$ is again an external point of $\mathcal{Q}$ since

$$
\sigma\left(G_{0}, Q_{1}\right) \leq \sigma\left(G_{0}, P_{1}\right)+\sigma\left(P_{1}, Q_{1}\right)<\frac{2 \rho}{3}+\frac{\rho}{3}=\rho .
$$


Likewise we are able to see that $Q_{2}:=P_{2}^{\tau}$ and $Q_{3}:=P_{3}^{\tau}$ are external points. The lines $q_{j}:=p_{j}^{\tau}(j \in\{1,2,3\})$ are secants of $\mathcal{Q}$, since they carry internal points.

Now we have found a non-trivial Desargues configuration with the required properties.

The property of being a secant line of $\mathcal{Q}$ does not change when we apply $\varphi$. Furthermore, $\varphi$ maps external points $A \in g$ to external points $A^{\varphi} \in g^{\varphi}$ (see (4)). Therefore $\varphi$ maps the Desargues configuration from above onto a Desargues configuration with the same properties. But $\mathcal{P}$ is a Desarguesian plane, so that $G^{\varphi}, H^{\varphi}$, and $I^{\varphi}$ are again collinear.

It remains to be shown that $G^{\varphi} H^{\varphi}$ is an external line of $\mathcal{Q}$. Every two orthogonally intersecting lines determine an external line. On every external line there is a pair of points $A, B$ such that $A^{\pi}$ intersects $B^{\pi}$ orthogonally. Since $\varphi$ maps orthogonally intersecting lines onto orthogonally intersecting lines, the $\varphi$-images of external lines are again external lines.

Now we achieved the aim of this paper. Using Proposition 7, we can show Proposition 4 and 5 in exactly the same way as we did above. Altogether we get an extension of Proposition 5:

Theorem 1. Let $\varphi$ be a bijection satisfying

$$
\bar{a} \sim \bar{b} \Longrightarrow \bar{a}^{\varphi} \sim \bar{b}^{\varphi} \quad \forall \bar{a}, \bar{b} \in \overline{\mathcal{G}} .
$$

in a hyperbolic space $\Pi_{h}$ with $\operatorname{dim} \Pi_{h} \neq 3$. Then $\varphi$ is induced by a $\mathcal{Q}$-collineation $\psi$ of $\Pi$. Moreover, $\varphi$ is a Plücker transformation.

Remark. Plücker transformations in hyperbolic spaces with $\operatorname{dim} \Pi_{h}=3$ cannot be investigated with the methods introduced in this paper. In Proposition 3 the crucial property of $\mathcal{H}$ is $\operatorname{dim} \Pi_{h} \geq 4$. In section 4.1 and 4.2 we use more than once that hyperbolic spaces with $\operatorname{dim} \Pi_{h}=2$ are the only ones in which no skew lines exist. Moreover we use that two intersecting lines do not have a common orthogonal line. Therefore we will have to use completely different

methods for the 3-dimensional case, which will be discussed in a forthcoming paper.

\section{References}

[1] Brauner H.: Geometrie projektiver Räume I, B.I. Wissenschaftsverlag, Mannheim Wien Zürich, 1976.

[2] Brauner H.: Geometrie projektiver Räume II, B.I. Wissenschaftsverlag, Mannheim Wien Zürich, 1976.

[3] Benz W.: Geometrische Transformationen, B.I. Wissenschaftsverlag, Mannheim Leipzig Zürich, 1992. 
[4] Benz W., SchröDer E.M.: Bestimmung der orthogonalitätstreuen Permutationen euklidischer Räume, Geom. Dedicata 21, 265 - 276, 1986.

[5] Chow W.L.: On the geometry of algebraic homogeneous spaces, Ann. of Math. 50, 32 - 67, 1949.

[6] Frank R.: Ein lokaler Fundamentalsatz für Projektionen, Geometriae Dedicata 44, 53 - 66, 1992.

[7] FRIEDLEIN H.-R.: Normalformen für Bewegungen in hyperbolischen Räumen, J. Geom. 23, $61-71,1984$.

[8] Giering O.: Vorlesungen über höhere Geometrie, Vieweg, Braunschweig/ Wiesbaden, 1982.

[9] Gudder S.: Metric Spaces over ordered Fields, Demonstr. Math. 19, No. 1, $165-183,1996$.

[10] Havlicek H.: On Plücker Transformations of generalized elliptic spaces, Rend. Mat. Appl., VII Ser. 14, 39 - 56, 1994.

[11] Havlicek H.: Symplectic Plücker Transformations, Math. Pannonica 6, $145-153,1995$.

[12] HöFER R.: Kennzeichnungen hyperbolischer Bewegungen durch Lineationen, J. Geom. 61, 56-61, 1998.

[13] Huang W.-L.: Adjacency Preserving Transformations of Grassmann Spaces, Abh. Math. Sem. Univ. Hamburg (in print).

[14] Kroll H.-J., Sörensen K.: Hyperbolische Räume, J. Geom. 61, 141 $149,1998$.

[15] Lester J.: On Distance Preserving Transformations of Lines in Euclidean Three-Space, Aequat. Math. 18, 69 - 72, 1985.

[16] LenZ H.: Vorlesungen über projektive Geometrie, Akademische Verlagsgesellschaft Geest \& Portig K.-G., Leipzig, 1965.

[17] Wif̧SŁAW W.: Topological Fields, Marcel Dekker, Inc., New York-Basel, 1988.

Anschrift des Autors: K. List, Institut für Geometrie, TU Wien, Wiedner Hauptstraße 8-10, A-1040 Wien 